Thorax (1960), 15, 165.

\title{
CAVO-PULMONARY ANASTOMOSIS
}

\author{
BY \\ B. J. BICKFORD AND F. RONALD EDWARDS \\ From the Department of Thoracic Surgery, University of Liverpool, and the \\ Royal Liverpool Children's Hospital
}

(RECEIVED FOR PUBLICATION JANUARY 6, 1960)

In some forms of cyanotic congenital heart disease ultimate direct surgical correction may be technically impossible. Tricuspid atresia, transposition of the great vessels with pulmonary stenosis, and the cyanotic group of patients with dextrocardia and multiple abnormalities are examples.

The classical systemic-pulmonary arterial anastomosis as described by Blalock and Potts, and the pleural strip described by Barrett, may produce some improvement in the pulmonary circulation in such cases, but usually this is small and the clinical result is disappointing. Furthermore a systemic-pulmonary anastomosis may add to the strain on the left ventricle.

A direct anastomosis between the superior vena cava and the right pulmonary artery will allow some $40 \%$ of the total venous return to pass directly through the lungs without any ventricular action, and for the purpose of providing palliative treatment for the above conditions might offer some advantages over the systemic-pulmonary shunts. The operation can be performed with relative ease in small children, on whom an arterial anastomosis may be difficult and may rapidly become inefficient due to the increased oxygen demands occurring with growth.

Experimental work on both sides of the Atlantic by Glenn and Patiño (1954), Patiño, Glenn, Guilfoil, Hume, and Fenn (1955), Robicsek, Temesvári, and Kádár (1956), Robicsek, Magistro, Foti, Robicsek, and Sanger (1958), Nuland, Glenn, and Guilfoil (1958), together with work done in Russia from 1951 onwards and reported by Bakulev and Kolesnikov (1959), has shown that anastomosis of the superior vena cava to the right pulmonary artery is fully compatible with survival in the experimental animal, the vena cava being divided above its entry into the right atrium and the right pulmonary artery severed as near as possible to its origin from the main pulmonary trunk.
Certain limitations are evident:

(1) The diameter of the right pulmonary artery should be such that it will transmit the caval flow without obstruction. Its diameter should be at least two-thirds that of the superior vena cava, giving an effective area of approximately half that of the superior vena cava. This is a theoretical concept, and may require revision in the light of further experience. An angiocardiogram is thus essential to ensure that this knowledge is available before the operation is undertaken.

(2) An increased pulmonary vascular resistance must not be present, as the superior vena caval pressure would have to rise to a high level to force the blood through the pulmonary vasculature, with possible secondary effects upon the cerebrum. What venous pressure the cerebrum is able to withstand permanently is unknown. In cases of constrictive pericarditis, a venous pressure of $30 \mathrm{~mm} . \mathrm{Hg}$ which has persisted for a long period without evidence of brain dysfunction is frequently encountered. At the end of the operation of cavo-pulmonary anastomosis with lungs of normal vascular resistance the superior vena caval pressure is approximately $25 \mathrm{~mm}$. $\mathrm{Hg}$. Our experience has shown that this is not associated with any encephalographic changes and the patient wakes up normally. Thus it is important that the pulmonary arterial pressure and pulmonary vascular resistance should be known before the operation is definitely decided upon.

(3) The presence of a left-sided superior vena cava should be determined. If the operation is performed on the right side, an effective flow through the right pulmonary arterial system would be jeopardized by blood escaping through the leftsided venous return. If no angiographic evidence of a left-sided superior vena cava is present it should be sought for at the time of the operation by clamping the superior vena cava while continuous pressure recordings and E.E.G. tracings are taken. If the pressure in the right superior vena cava does not rise markedly and the E.E.G. 
tracings remain normal, then the presence of a left superior vena cava must be deduced and this will require ligation either at the same operation or subsequently. Complete obstruction of the superior vena cava when it is the only venous return from the brachio-cephalic system causes a rise in pressure to $80-90 \mathrm{~mm}$. $\mathrm{Hg}$ within 10 seconds, and a rapid onset of the high-voltage, low-frequency rhythm in the E.E.G. characteristic of venous obstruction.

\section{Clinical Application}

The first clinical record of the use of cavopulmonary anastomosis in the literature in English is that of Glenn (1958). The patient was a boy of 7 , with tricuspid atresia, transposition of the great vessels, and pulmonary stenosis. The clinical result of the operation was extremely good. Sanger, Robicsek, and Taylor (1959) reported a further successful operation, in the case of an 11year-old boy with transposition of the great vessels, an atrial septal defect, and pulmonary stenosis.

Bakulev and Kolesnikov (1959) describe the results of cavo-pulmonary anastomosis performed on 41 patients with cyanotic heart disease irrespective of the type. Sixteen patients survived the operation, with 14 good results. They mention that the first successful clinical application of the method had been obtained by Meshalkin in 1956 and that they themselves had first performed a cavo-pulmonary anastomosis in a patient in 1951.

In his original description Glenn (1958) described the essentials of the technique which we have used, but Bakulev and Kolesnikov (1959) favoured a direct end-to-end anastomosis between the superior vena cava and the pulmonary artery. This has certain disadvantages. It is clear from their studies that complete clamping of the superior vena cava quickly produces changes in the brain together with cyanosis, facial oedema, exophthalmos, petechial haemorrhages on the face and the upper half of the body, as well as a lymphatic discharge from the nose. Oedema and other signs disappear within 24 hours after release of the compression. The Russian workers used the blood vessel stitcher which has been developed in their country and by this means were able to cut down the time of occlusion of the superior vena cava to five or six minutes. We feel that a similar result can be obtained without the disadvantage of total occlusion of the superior vena cava by using Glenn's method of end-to-end anastomosis of the pulmonary artery to the caval vessel followed by division of the superior vena cava itself below the anastomosis. After this division the vessels imme- diately assume what is virtually a straight line for $\stackrel{\overrightarrow{\bar{B}}}{\rightarrow}$ discharge of blood from the caval system into the lung.

\section{TEChNique OF OPERATION}

This is illustrated in Fig. 1.

A right-sided thoracotomy is performed through के an antero-lateral incision in the fourth interspace. $\vec{O}$ The pericardium is opened and the relevant structures $\overrightarrow{\vec{A}}$ examined. The superior vena cava is dissected from $\vec{\omega}$ the front of the pulmonary artery. The right pulmonary artery pressure is checked both before and $\vec{x}$ after compression to ensure that the flow through $\vec{\sim}$ the left lung is satisfactory. The superior vena caval of pressures are then taken before and after compression just above the azygos vein. If the pressure rises $\vec{G}$ rapidly to $50 \mathrm{~mm}$. $\mathrm{Hg}$ then a pressure sufficient to maintain a pulmonary circulation can be guaranteed. $\triangle$ If the pressure rises not at all, or only a few milli- $\vec{c}$ metres of mercury, then there must be an alternative $\subseteq$ return to the heart, probably a left-sided superior vena cava. When this is the case the operation will $\vec{\theta}$ have to be extended to ligate this structure. The right $\mathscr{O}$ pulmonary artery is dissected right back to the com-. mon pulmonary artery trunk and ligated, transfixed, and divided at this level. Control of the reflux from the lungs is maintained by double encircling ligatures on the pulmonary artery branches. The cut end of the pulmonary artery must not be damaged by clamp $\mathbb{D}$ or ligature.

A lateral clamp (Beck's aortic clamp) is put on $\overrightarrow{0}$ to the superior vena cava so that its lowest edge allows 3 about $2 \mathrm{~cm}$. of free vessel before its insertion into the left atrium. The position of this clamp will be decided by the position at which the cut end of the pulmonary artery lies most comfortably. A careful $\vec{\circ}$ watch is kept on the superior vena cava to see that the pressure does not rise after tightening the clamp. Electro-encephalographic control gives the best $\frac{5}{3}$ indication of this, and any alteration in the tracing should be reported to the operating team so that the position of the clamp may be altered.

An incision in the superior vena cava, correspond-응 ing in length with the flattened end of the pulmonary artery, is made and then the two structures are sutured together with 4/0 silk. A continuous everting suture on each side is used. After completion of the $N$ anastomosis the double circle ligatures on the pulmonary artery branches are released to allow some $\mathcal{O}$ reflux of blood to seal the stitch holes. Finally the $\mathbb{N}$ caval clamp is removed, and the caval blood should fill the pulmonary artery. When the anastomosis is seen to be satisfactory the portion of the vena cava

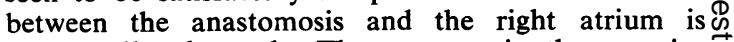
temporarily clamped. The pressure in the superior vena cava should not then rise above $25 \mathrm{~mm}$. Hg. $\frac{0}{0}$ Finally this portion of the vena cava is doubly $\frac{\mathrm{D}}{\mathbb{D}}$ ligated, transfixed, and divided, when the upper part $\stackrel{?}{\mathcal{P}}$ of the vena cava will slip upwards and rotate so that $\stackrel{\mathbb{Q}}{\mathscr{Q}}$ the vena cava and the pulmonary artery appear to lie in linear continuity.

The chest is then closed with drainage. 


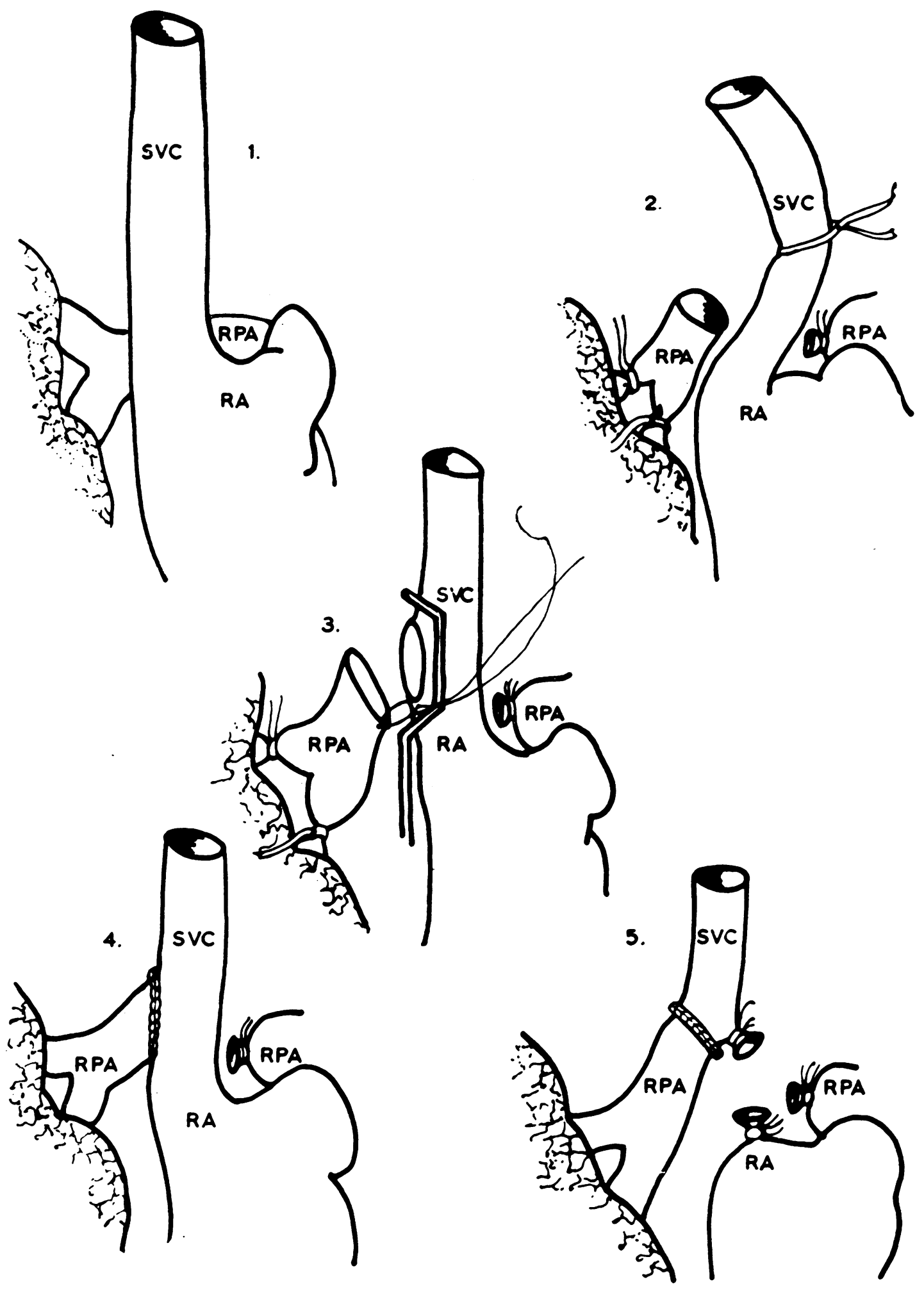

FIG. 1.-Technique of cavo-pulmonary anastomosis (after Glenn). 1, Normal anatomy; 2, the right pulmonary artery is divided; 3, the superior vena cava is clamped laterally, incised, and the anastomosis started; 4 , the anastomosis completed; 5 , the superior vena cava is divided between the anastomosis and the right atrium. 


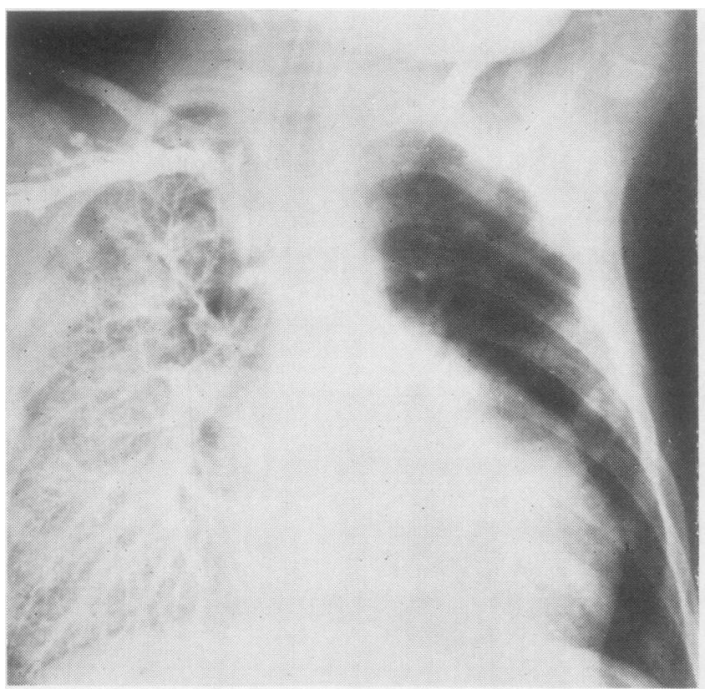

FIG. 2.-Angiogram taken six months after cavo-pulmonary anastomosis for tricuspid atresia showing direct communication between superior vena cava and right pulmonary artery (Case 1).

\section{RESULTS}

We have performed a cavo-pulmonary anastomosis by the technique described in two girls aged 6 and 7 years suffering from tricuspid atresia. Both had had left-sided Blalock systemic-pulmonary anastomosis performed at the age of 2 . There had been some relief of the cyanosis and dyspnoea for two to three years after this procedure, but recently both had become more incapacitated, with severe "cyanotic attacks" and marked dyspnoea. Convalescence was uneventful in both cases, and they were discharged home on the twelfth day after operation.

These two children have been followed up for six months and two months respectively. Both are now much pinker and their parents describe them as "very active" with dyspnoea only on marked exertion. A trace of cyanosis is visible in both, however. The post-operative haemoglobin level has fallen in one child from $154 \%$ pre-operatively to $110 \%$ (R.B.C.s 5.8 million), and in the other from $112 \%$ to $83 \%$ (R.B.C.s 4.5 million).
A noticeable feature is that both childre become distinctly more cyanotic on lying downe indicating that there is a considerable gravitationat supplement to the flow through the lungs. The do not complain of any congested feeling in the face. The external jugular veins are visible in the्छ erect position and more so on lying down, but there is no pulsation. Both children can be con:sidered to be remarkably improved, and would appear to be able to undertake a normal life, a any rate for the time being. The angiogran shown in Fig. 2 was taken six months after the operation on the first child, and shows excellents filling of the right pulmonary arterial system fron the anastomosed superior vena cava.

\section{SUMMARY}

Anastomosis between the superior vena cavæ and the right pulmonary artery can be utilized to relieve the cyanosis and dyspnoea in irreparables forms of congenital heart disease.

Its use is particularly applicable to tricuspi申 atresia.

An account of the technique is described ant the results of operation in two cases of tricuspig atresia given.

We would like to thank Dr. Gordon Farquhar fo presenting these two cases to us for surgical treato ment, and to Dr. G. Jackson Rees for the electroo encephalographic studies.

\section{REFERENCES}

Bakulev, A. N., and Kolesnikov, S. A. (1959). J. thorac. Surg., 3ட் 693.

Barrett, N. R., and Daley, R. (1949). Brit. med, J., 1, 699.

Blalock, A and Taussig H B (1945). J.Amer med As 128,189

Glenn, W. W. L. (1958). New Engl. J. Med., 259, 117

- and Patiño. J. F. (1954). Yale J. Biol. Med., 27, 147

Meshalkin, E. N. (1956). Eksp. Hir., 1, No. 6, p. 3. (Cited Bakulev and Kolesnikov, supra.)

Nuland, S. B., Glenn, W. W. L., and Guilfoil, P. H. (1958). Surger

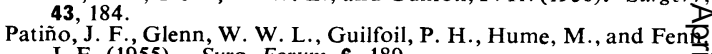
J. E. (1955). Surg. Forum, 6, 189

Potts, W. J., Smith, S., and Gibson, S. (1946). J. Amer. med. As. Av

132, 627.
Robicsek, F., Magistro, R., Foti, E., Robicsek, L., and Sanger, P. W (1958). J. thorac. Surg, 35, 440. 151

Sanger, P. W., Robicsek, F., and Taylor, F. H. (1959). J. thoraf Cardiovasc. Surg., 38, 166. 